\title{
Bijective Recurrences concerning Schröder paths
}

\author{
Robert A. Sulanke \\ Boise State University \\ Boise, Idaho, USA \\ sulanke@math.idbsu.edu
}

Submitted: April 7, 1998; Accepted: October 30, 1998

\begin{abstract}
Consider lattice paths in $\mathbf{Z}^{2}$ with three step types: the up diagonal $(1,1)$, the down diagonal $(1,-1)$, and the double horizontal $(2,0)$. For $n \geq 1$, let $S_{n}$ denote the set of such paths running from $(0,0)$ to $(2 n, 0)$ and remaining strictly above the x-axis except initially and terminally. It is well known that the cardinalities, $r_{n}=\left|S_{n}\right|$, are the large Schröder numbers. We use lattice paths to interpret bijectively the recurrence $(n+1) r_{n+1}=3(2 n-1) r_{n}-(n-2) r_{n-1}$, for $n \geq 2$, with $r_{1}=1$ and $r_{2}=2$.

We then use the bijective scheme to prove a result of Kreweras that the sum of the areas of the regions lying under the paths of $S_{n}$ and above the x-axis, denoted by $A S_{n}$, satisfies $A S_{n+1}=6 A S_{n}-A S_{n-1}$, for $n \geq 2$, with $A S_{1}=1$, and $A S_{2}=7$. Hence $A S_{n}=1,7,41,239,1393, \ldots$ The bijective scheme yields analogous recurrences for elevated Catalan paths.

Mathematical Reviews Subject Classification: 05A15
\end{abstract}

\section{The paths and the recurrences}

We will consider lattice paths in $\mathbf{Z}^{2}$ whose permitted step types are the up diagonal $(1,1)$ denoted by $U$, the down diagonal $(1,-1)$ denoted by $D$, and the double horizontal $(2,0)$ denoted by $H$. We will focus on paths that run from $(0,0)$ to $(2 n, 0)$, for $n \geq 1$, and that never touch or pass below the x-axis except initially and terminally. Let $C_{n}$ denote the set of such paths when only U-steps and D-steps are allowed, and let $S_{n}$ denote the set of such paths when all three types are allowed. It is well known that the cardinalities $c_{n}=\left|C_{n}\right|$ and $r_{n}=\left|S_{n}\right|$, for $n \geq 1$, are the Catalan numbers and the large Schröder numbers, respectively. (See Section 4, particularly Notes 2 and 4.) Hence, here one might view the elements of $S_{n}$ as elevated Schröder paths. Let $A C_{n}$ denote the sum of the areas of the regions lying under the paths of $C_{n}$ and 
above the x-axis. Likewise, let $A S_{n}$ denote the sum of the areas of the regions lying under the paths of $S_{n}$ and above the x-axis.
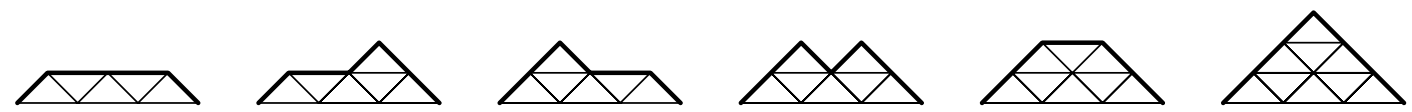

Figure 1: The 6 elevated Schröder paths of $S_{3}$ bound 41 triangles of unit area.

\begin{tabular}{r|rrrrrl}
$n$ & 1 & 2 & 3 & 4 & 5 & $\ldots$ \\
\hline$c_{n}$ & 1 & 1 & 2 & 5 & 14 & $\ldots$ \\
$r_{n}$ & 1 & 2 & 6 & 22 & 90 & $\ldots$ \\
$A C_{n}$ & 1 & 4 & 16 & 64 & 256 & $\ldots$ \\
$A S_{n}$ & 1 & 7 & 41 & 239 & 1393 & $\ldots$
\end{tabular}

The Catalan numbers and the Schröder numbers have been studied extensively; Section 4 references some studies related to lattice paths. In our notation their explicit formulas are, for $n \geq 1$,

$$
c_{n}=\frac{1}{n}\left(\begin{array}{c}
2 n-2 \\
n-1
\end{array}\right) \quad \text { and } \quad r_{n}=\sum_{k=1}^{n} \frac{1}{k}\left(\begin{array}{l}
n-2 \\
k-1
\end{array}\right)\left(\begin{array}{c}
n-1 \\
k-1
\end{array}\right) 2^{k} .
$$

It is known that these sequences satisfy the recurrences

$$
\begin{aligned}
(n+1) c_{n+1} & =2(2 n-1) c_{n} \\
(n+1) r_{n+1} & =3(2 n-1) r_{n}-(n-2) r_{n-1}
\end{aligned}
$$

for $n \geq 2$, with initial conditions $c_{1}=1, c_{2}=1, r_{1}=1$, and $r_{2}=2$.

We will give a bijective proof for (1) and (2) when the sequences $c_{n}$ and $r_{n}$ are defined in terms of the sets of lattice paths. We will then employ this bijective construction to obtain a combinatorial interpretation that the sequences for the total areas satisfy

$$
\begin{aligned}
A C_{n+1} & =4 A C_{n} \\
A S_{n+1} & =6 A S_{n}-A S_{n-1}
\end{aligned}
$$

for $n \geq 2$ with initial conditions $A C_{1}=1, A C_{2}=4, A S_{1}=1$, and $A S_{2}=7$.

Using binary trees, Rémy [10] gave a combinatorial proof of recurrence (1). Recently, Foata and Zeilberger [3] showed bijectively, using well-weighted binary plane trees, that the small Schröder numbers satisfy (2) with initial conditions $r_{1}=1$ and $r_{2}=1$. (See Section 4 for "well-weighted" and "small".) Kreweras [4], using lattice 
paths equivalent to those of $S_{n}$ showed $A S_{n}=\sum_{0 \leq k<n} 2^{k}\left(\begin{array}{c}2 n-1 \\ 2 k\end{array}\right)$ and derived recurrence (4). Following his results, Bonin, Shapiro, and Simion [2] proved (4) using generating functions and then wrote that "This recurrence cries out for a combinatorial interpretation." Section 3 comes to the rescue.

\section{The proof of recurrences (1) and (2)}

We will focus on recurrence (2) rearranged as $3(2 n-1) r_{n}=(n+1) r_{n+1}+(n-2) r_{n-1}$. In $S_{n}$ replicate each path, defined as a sequence of steps, $3(2 n-1)$ times as follows: First repeatedly tag each path $P$ by appending the symbol $a, b$, or $c$. Next, for each tagged path $P$, consecutively index its steps, as positioned in $P$, with the integers 1 through $2 n-1$ so that each U-step and each non final D-step receives one integer and each H-step receives two consecutive integers. Then mark each path $P$ by selecting an integer from $\{1, \ldots, 2 n-1\}$ and marking the corresponding step on $P$

- by the superscript $x$ if the step is $U$ or if the step is $H$ with odd index, and

- by the superscript $y$ if the step is $D$ or if the step is $H$ with even index.

We write the set of such replications as $\{a, b, c\} \times\{1, \ldots, 2 n-1\} \times S_{n}=\{a, b, c\} \times$ $[2 n-1] \times S_{n}$, where, in general, $[n]$ denotes $\{1, \ldots, n\}$. For instance, for $S_{2}=$ $\{U U D D, U H D\}$,

$$
\begin{aligned}
& \{a, b, c\} \times[3] \times S_{2}= \\
& \left\{U^{x} U D D a, \quad U U^{x} D D a, \quad U U D^{y} D a, \quad U^{x} H D a, \quad U H^{y} D a, \quad U H^{x} D a,\right. \\
& U^{x} U D D b, \quad U U^{x} D D b, \quad U U D^{y} D b, \quad U^{x} H D b, \quad U H^{y} D b, \quad U H^{x} D b, \\
& \left.U^{x} U D D c, \quad U U^{x} D D c, \quad U U D^{y} D c, \quad U^{x} H D c, \quad U H^{y} D c, \quad U H^{x} D c\right\} .
\end{aligned}
$$

Next in $S_{n+1}$ replicate each path $n+1$ times by sequentially marking one of its U-steps or H-steps by the symbol $z$. This replicated set is denoted as $[n+1] \times S_{n+1}$. Similarly, in $S_{n-1}$ replicate each path $n-2$ times by sequentially marking one of its $\mathrm{H}$-steps or non final D-steps by the symbol $z$. This replicated set is denoted as $[n-2] \times S_{n-1}$.

For $n \geq 2$, we now define the desired bijection,

$$
f:\{a, b, c\} \times[2 n-1] \times S_{n} \rightarrow[n+1] \times S_{n+1} \bigcup[n-2] \times S_{n-1} .
$$

Suppose

$$
P=p_{1} \cdots p_{i} \cdots p_{j} \cdots p_{k} \cdots p_{m} .
$$

denotes a typical replicated path in $[2 n-1] \times S_{n}$ for which the following four items hold.

- The positions $i, j$, and $k$ satisfy $1 \leq i \leq j<k \leq m$. 
- The step $p_{j}$ is the step that is marked by $x$ or $y$.

- The step $p_{i}$ is the last U-step preceding $p_{j+1}$ for which $\operatorname{LEV}\left(p_{i}\right)=\operatorname{LEV}\left(p_{j}\right)$. Here the level of arbitrary step $p_{\ell}$, denoted $\operatorname{LEV}\left(p_{\ell}\right)$, is the ordinate of its final point. When $p_{j}=U^{x}, i=j$.

- The step $p_{k}$ is the first D-step after $p_{j}$ for which $\operatorname{LEV}\left(p_{k}\right)=\operatorname{LEV}\left(p_{j}\right)-1$.

Case 1a: If $p_{j}=U^{x}, H^{x}$, or $D^{y}, f(P a)=p_{1} \cdots p_{i} \cdots p_{j} U^{z} D p_{j+1} \cdots p_{k} \cdots p_{m}$.

(Here, $f(P a)$ is obtained by inserting the pair $U^{z} D$ immediately after $p_{j}$. The tags $x, y$, and $a$ are erased here; the tags $b$ and $c$ are erased in the following cases. If $P$ appears in Fig. 2, $f(P a)$ appears in Fig. 3. In the figures the dots pertain to an illustration for the proof of the next section.)

Case 1b: If $p_{j}=U^{x}, H^{x}$, or $D^{y}, f(P b)=p_{1} \cdots p_{i}^{z} \cdots p_{j} U R D p_{k} \cdots p_{m}$, where $R=$ $p_{j+1} \cdots p_{k-1}$.

(Observation 1: In the path $Q=q_{1} q_{2} \cdots q_{m+2}=f(P b)$ a D-step immediately precedes the D-step $q_{k+2}$, which is the translation of the step $p_{k}$. The step $q_{k+2}$ is the first step after $q_{i}^{z}=p_{i}^{z}$ for which $\operatorname{LEV}\left(q_{k+2}\right)=\operatorname{LEV}\left(q_{i}\right)-1 ; q_{j}=p_{j}$ is now the last step before $q_{k+2}$ such that $\operatorname{LEV}\left(q_{j}\right)=\operatorname{LEV}\left(q_{k+2}\right)-1$. The path $R$ may be empty.)

\begin{tabular}{|l|l|l|l|}
\hline If $P$ & $U U U D H^{x} H U D D D$ & $U H U H^{y} U H D D D$ & $U U U D H^{y} H U D D D$ \\
\hline then & & & \\
$f(P a)$ & $U U U D H \underline{U^{z} D} H U D D D$ & $U H U \underline{U}^{z} H \underline{D} U H D D D$ & $U U U D \underline{U^{z}} H \underline{D} U D D D$ \\
$f(P b)$ & $U U^{z} U D H \underline{H} H U D \underline{D} D D$ & $U H U^{z} H U H D \underline{H} D D$ & $U U^{z} U D \underline{U} H U D \underline{D} U D D D$ \\
$f(P c)$ & $U U U D H \underline{H^{z}} H U D D D$ & $U H U^{z} U H D H \underline{H} D D$ & $U U U D^{z} H U D D D$ \\
\hline
\end{tabular}

Table 1: This table spells out the examples of the Figures 1 to 9 and 11 to 14. Underlining identifies inserted steps.

Case 1c: If $p_{j}=U^{x}, H^{x}$, or $D^{y}, f(P c)=p_{1} \cdots p_{i} \cdots p_{j} H^{z} p_{j+1} \cdots p_{k} \cdots p_{m}$.

Case 2a: If $p_{j}=H^{y}, f(P a)=p_{1} \cdots p_{j-1} U^{z} H D p_{j+1} \cdots p_{m}$.

Case 2b: If $p_{j}=H^{y}, f(P b)=p_{1} \cdots p_{i}^{z} \cdots p_{j-1} U R D H p_{k} \cdots p_{m}$, where $R$ is the subpath $p_{j+1} \cdots p_{k-1}$.

(Here a U-step and a D-step are inserted and the step $p_{j}=H$ is moved. Observation 2: In the path $Q=f(P b)$ exactly one H-step immediately precedes $q_{k+2}$, the translation of $p_{k}$. The step $q_{j-1}=p_{j-1}$ is now the last step before $q_{k} q_{k+1} q_{k+2}=D H p_{k}$ such that $\operatorname{LEV}\left(q_{j-1}\right)=\operatorname{LEV}\left(q_{k+2}\right)+1 . R$ may be empty.) 
Case 2c: If $p_{j-1} p_{j}=U H^{y}, f(P c)=p_{1} \cdots p_{i}^{z} \cdots p_{j-1} R H H p_{k} \cdots p_{m}$, where $R$ is the subpath $p_{j+1} \cdots p_{k-1}$.

(Observation 3: In the path $Q=f(P c)$, at least two H-steps immediately precede the D-step $q_{k+1}$, the translation of $p_{k}$.)

Case 3: If $p_{j-1} p_{j}=H H^{y}$ or $D H^{y}, f(P c)=p_{1} \cdots p_{j-1}^{z} p_{j+1} \cdots p_{m}$.

(Here $f(P c) \in[n-2] \times S_{n-1}$ with the marked H-step being deleted.)

Table 1 and Figures 1 to 9 and 11 to 14 illustrate the map $f$. By giving special attention to the three Observations in the preceding, it is straight forward to check the necessary cases to show that $f$ is a bijection. Assigning cardinalities to the sets in the bijection given in (5) yields the recurrence (2). To prove recurrence (1), simply remove all reference to the $\mathrm{H}$-steps and to the $\operatorname{tag} c$ in the proof.

\section{The proof of recurrences (3) and (4)}

Retaining the previous notions, consider the recurrence (4). One can partition the region under a path and above the x-axis by copies of two isosceles right triangles whose hypotenuses have length 2 and are parallel to the x-axis. Figure 1 illustrates how these triangles of unit area uniquely partition the regions under the paths. A triangle is called an up triangle if its right angle is above its hypotenuse; otherwise, it is called a down triangle.

An up-triangle-strip (down-triangle-strip, respectively) under a path of $S_{n}$ is a maximal array of up (down, respectively) triangles having the centers of their hypotenuses on a single line of slope -1 (slope 1, respectively). It is easily seen that each path in $S_{n}$ has $n$ up-triangle-strips and $n-1$ down-triangle-strips. The marked triangles in Figure 2 illustrate an up-triangle-strip; those in Figure 6 illustrate a down-triangle-strip. Each marked path $P \in[2 n-1] \times S_{n}$ determines a unique strip under $P$ as follows: If the step $p_{j}$ is marked by $x$, then the corresponding strip is the up-triangle-strip whose line of centers of its triangles intersects the step $p_{j}$. If $p_{j}$ is marked by $y$, then the corresponding strip is the down-triangle-strip whose line of centers of its triangles intersects the step $p_{j}$. In either case we designate by $6 T_{P}$ six copies of the strip corresponding to the step $p_{j}$.

In the region under any path in $S_{n+1}$ a contiguous-strip is a maximal array of up and down triangles having the centers of their hypotenuses on a single line of slope -1. Each marked path $P \in[n+1] \times S_{n+1}$ determines a unique strip under $P$, namely that contiguous-strip whose line of hypotenuse centers intersects the marked step of $P$. We designate this strip by $T C_{P}$. The marked triangles of Figure 3 indicate a contiguous-strip. 


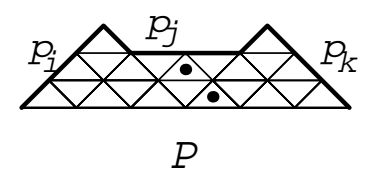

Fig.2

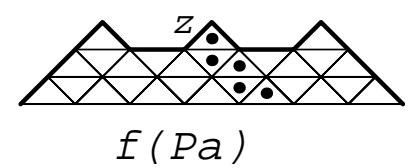

Fig. 3

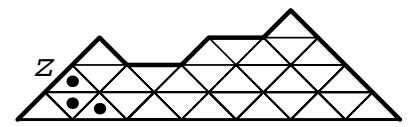

$f(P b)$

Fig. 4

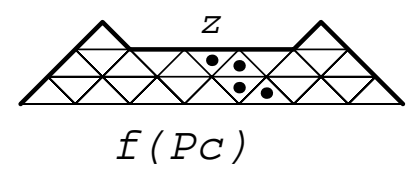

Fig. 5

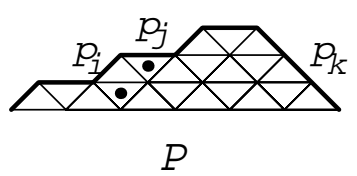

Fig. 6

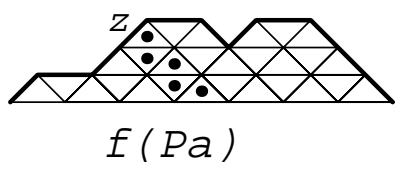

Fig. 7

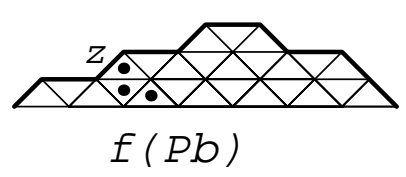

Fig. 8

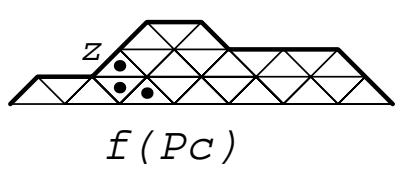

Fig. 9

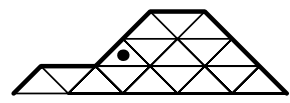

isolated-triangle

Fig. 10

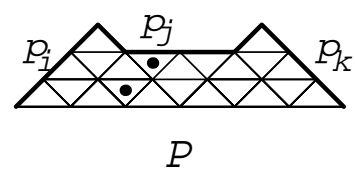

Fig.11

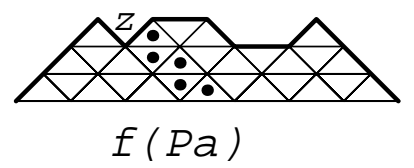

Fig. 12

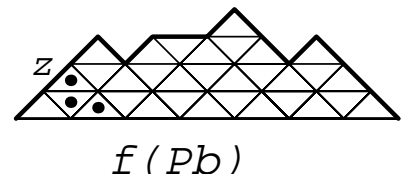

Fig. 13

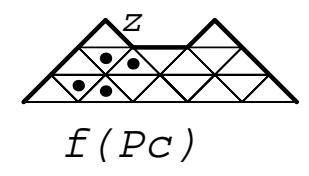

Fig. 14

Figures 2 through 14. 
In the region under any path in $S_{n-1}$ each down triangle can always be paired with the contiguous up triangle on its right, but not visa-versa. A diamond-strip is a maximal array of such pairs of triangles whose common sides lie on a line of slope 1 . The marked step of each path $P \in[n-2] \times S_{n-1}$ determines a unique diamond-strip under $P$, namely the diamond-strip whose line of common sides intersects the final point of the z-marked step of $P$. We designate this diamond-strip by $T D_{P}$. The marked triangles of Figure 14 indicate a diamond-strip.

Under a path $P \in[n-2] \times S_{n-1}$, any up triangle that is not contiguous along its left side with a down triangle is viewed as an isolated-triangle. Figure 10 illustrates an isolated-triangle. Since the left side of each isolated-triangle is a U-step of $P$ and conversely, each U-step, say $p_{h}$, uniquely matches an isolated-triangle that we designate by $T I_{P, h}$. The disjoint collection of strips and isolated-triangles

$$
\left(\bigcup_{P \in[n-2] \times S_{n-1}}\left\{T D_{P}\right\}\right) \bigcup\left(\bigcup_{P \in S_{n-1}} \bigcup_{h \in u(P)}\left\{T I_{P, h}\right\}\right)
$$

partitions the total region under the paths in $S_{n-1}$, where $u(P)$ is the set of the positions of the U-steps on $P$.

To construct a function that yields a combinatorial proof of recurrence (4), consider defining

$$
g: \bigcup_{P \in[2 n-1] \times S_{n}}\left\{6 T_{P}\right\} \rightarrow \mathcal{T} \bigcup \mathcal{Q}
$$

where the elements of $\mathcal{T}$ and $\mathcal{Q}$ will be ordered triples and ordered quadruples, respectively, of mutually non overlapping strips partitioning the total region lying under the paths of $S_{n+1}$ and $S_{n-1}$.

With $P$ being an arbitrary path as in (6), the bijection $f$ induces a function $g$ so that

Case i: if $p_{j}=U^{x}, H^{x}$, or $D^{y}$, define

$$
g\left(6 T_{P}\right)=\left(T C_{f(P a)}, T C_{f(P b)}, T C_{f(P c)}\right) .
$$

Case ii: if $p_{j-1} p_{j}=p_{i} p_{j}=U H^{y}$, define

$$
g\left(6 T_{P}\right)=\left(T C_{f(P a)}, T C_{f(P b)}, T C_{f(P c)}, T I_{R, i}\right)
$$

where $R=p_{1} \cdots p_{i} p_{i+2} \cdots p_{m}$.

Case iii: if $p_{j-1} p_{j}=H H^{y}$ or $D H^{y}$, define

$$
g\left(6 T_{P}\right)=\left(T C_{f(P a)}, T C_{f(P b)}, T D_{f(P c)}\right) .
$$

The mapping of six copies of the strip of Figure 2 to those of Figures 3 to 5 illustrates Case i. Likewise Figure 6 with Figures 7 to 10 illustrates Case ii, and Figure 11 with Figures 12 to 14 illustrates Case iii. Notice that each column of the array of figures shows the 6 -fold transfer of area. 
The function $f$ being bijective implies $g$ is bijective. The following three items can be routinely checked to show that $g$ transfers area as claimed. Here $\mathrm{A}(T)$ denotes the area of an arbitrary strip $T$. For

Case i, $\left(\mathrm{A}\left(T C_{f(P a)}\right), \mathrm{A}\left(T C_{f(P b)}\right), \mathrm{A}\left(T C_{f(P c)}\right)\right)=\left(2 \mathrm{~A}\left(T_{P}\right)+1,2 \mathrm{~A}\left(T_{P}\right)-1,2 \mathrm{~A}\left(T_{P}\right)\right)$;

Case ii, $\left(\mathrm{A}\left(T C_{f(P a)}\right), \mathrm{A}\left(T C_{f(P b)}\right), \mathrm{A}\left(T C_{f(P c)}\right), \mathrm{A}\left(T I_{R, j}\right)\right)=\left(2 \mathrm{~A}\left(T_{P}\right)+1,2 \mathrm{~A}\left(T_{P}\right)-\right.$ $\left.1,2 \mathrm{~A}\left(T_{P}\right)-1,1\right)$

Case iii, $\left(\mathrm{A}\left(T C_{f(P a)}\right), \mathrm{A}\left(T C_{f(P b)}\right), \mathrm{A}\left(T D_{f(P c)}\right)\right)=\left(2 \mathrm{~A}\left(T_{P}\right)+1,2 \mathrm{~A}\left(T_{P}\right)-1,2 \mathrm{~A}\left(T_{P}\right)\right)$;

Finally to prove recurrence (3) we remove all reference to $\mathrm{H}$-steps and the tag $\mathrm{c}$ in this proof.

\section{Notes}

1. The following corollary of the construction of the function $f$ originated as a fortuitous observation resulting in the definition for the crucial Case 3 :

For $n \geq 2$, there are $(n-2) r_{n-1}$ step pairs of the form $D H$ or $H H$ on the totality of paths of $S_{n}$.

2. One of the more interesting of the many references to the Catalan numbers is Stanley's [15] collection of 66 combinatorial interpretations of these numbers. His book [15] lists other primary references in the vast literature for these numbers.

3. In lieu of the three step types employed in this paper, the step types $(0,1)$, $(1,0)$, and $(1,1)$ are the usual step types defining Schröder paths. For the latter three types, clearly the Schröder number $r_{n}$ counts the paths running from $(0,0)$ to $(n-1, n-1)$ and never passing below the line $y=x$. In an early paper on paths with such step types Moser and Zayachkowski [7], realizing that the number of unrestricted paths from $(0,0)$ to $(n, n)$ is a Legendre polynomial evaluated at 3 , used a recurrence for these polynomials to derive essentially recurrence (2).

4. We use " $r_{n}$ " for the large (or double as in [4]) Schröder numbers since $s_{n}=$ $r_{n} / 2$ for $n \geq 2$ with $s_{1}=1$ is reserved for the so-called small Schröder numbers: $1,1,3,11,45, \ldots$. Ernst Schröder formulated these numbers in the second problem of his 1870 paper [14]: In how many ways can one or more pairs of brackets be legally placed in $z_{1}, z_{2} \cdots z_{n}$ ? For instance, when $n=3$, there are the three bracketings, $\left(z_{1} z_{2} z_{3}\right),\left(\left(z_{1} z_{2}\right) z_{3}\right)$, and $\left(z_{1}\left(z_{2} z_{3}\right)\right)$. The problem of enumerating bracketings is equivalent both to the problem of enumerating dissections of convex polygons and to the problem of enumerating Schröder trees with a fixed number of leaves. (A Schröder tree is a plane trees whose internal nodes have at least two children.)

As noted in [16], David Hough discovered that the small Schröder numbers were apparently known to Hipparchus in the second century B.C. as counting certain logical propositions. The papers $[2,9,12,11,13,16,17]$ form a selection of the studies concerning the Schröder numbers. Of particular interest is the result of Rogers and Shapiro, appearing implicitly in $[12,13]$, and later the result of Bonin, Shapiro, and 
Simion [2], that give combinatorial maps relating the enumeration of bracketings to the enumeration of lattice paths of $S_{n}$.

5. A well-weighted binary plane tree is a binary tree where each node having a right internal child is labeled with a 1 or a 2. Foata and Zeilberger [3], after giving a rather simple bijection between Schröder trees and well-weighted binary plane trees, showed bijectively that the small Schröder numbers satisfy $(n+1) s_{n+1}=$ $3(2 n-1) s_{n}-(n-2) s_{n-1}$ with the conditions $s_{1}=1$, and $s_{2}=1$. Their proof is not isomorphic to our proof of (2); this is not surprising since the bijections mentioned at the end of Note 3 between bracketings and $S_{n}$ do not seem to be trivial.

6 . In this and the next note define sequence $A S_{n}$ purely as the one satisfying the formal recurrence (4), not specifically in terms of lattice paths. Barcucci, Brunetti, Del Lungo, and Del Rietoro [1] recently gave a combinatorial interpretation of formal recurrence (4) in terms of a regular language. In [5] the sequence $A S_{n}$ is related to solutions of the diophantine equation, $x^{2}+(x+1)^{2}=y^{2}$, with $x=\left(A S_{n}-1\right) / 2$. Newman, Shanks, and Williams [8] found that the numbers $A S_{n}$ correspond to the orders of certain simple groups.

7. The author [18] has considered the formal recurrences (1) to (4) bijectively in terms of parallelogram polyominoes. For $n \geq 2$, let $p_{\alpha, n}(w)=\sum_{k} p_{\alpha, n, k} w^{k}$, where $p_{0, n, k}$ denotes the number of parallelogram polyominoes with perimeter $2 n$ and width $k$, and where $(n-1) p_{1, n, k}$ denotes the total area of such polyominoes. The paper [18] shows that the sequences $p_{0, n}(w)$ and $p_{1, n}(w)$ satisfy the recurrences

$$
(n+1-\alpha) p_{\alpha, n+1}(w)=(2 n-1-\alpha)(1+w) p_{\alpha, n}(w)-(n-2)(1-w)^{2} p_{\alpha, n-1}(w)
$$

with initial conditions $p_{\alpha, 2}(w)=w, p_{\alpha, 3}(w)=w+w^{2}$. The proof for the case $\alpha=0$ in [18] is isomorphic to the proof in [3], but not to the proof of Section 2.

More specifically, the total area $(n-1) p_{1, n}(1)$ is $4^{n-2}$; this result was recently derived by interesting generating-function argument by Woan, Shapiro, and Rogers [19]. The product $(n-1) p_{1, n}(2)$, corresponding to the sum of the areas of polyominoes having bi-colored columns, satisfies the recurrence $n p_{1, n+1}(2)=6(n-1) p_{1, n}(2)-$ $(n-2) p_{1, n-1}(2)$ with early values $(n-1) p_{1, n}(2)=1,6,35,204,1189, \ldots$, for $n=$ $2,3,4,5,6 \ldots$ These polynomial sequences, $p_{\alpha, n}(w)$, generalize other well-known sequences: e.g., $\left\{p_{0, n}(1)\right\}_{n \geq 2}$ are the Catalan numbers, $\left\{p_{0, n}(2)\right\}_{n \geq 2}$ are the large Schröder numbers, $\left\{\left((n-1) p_{1, n}(2) / 2\right)^{2}\right\}_{n \geq 2}$ are the square-triangular numbers, and $\left\{p_{2, n}(1)\right\}$ are the central binomial coefficients.

8. Recently, Merlini, Sprugnoli, and Verri [6] used generating function methods in determining the sum of the areas bounded by constrained lattice paths belonging to sets that essentially generalize $C_{n}$ and $S_{n}$. The paper [6] also contains additional relevant references to the literature. 
THE ELECTRONIC JOURNAL OF COMBINATORICS 47 (1998) \#R47

\section{References}

[1] E. Barcucci, S. Brunetti, A. Del Lungo, and F. Del Rietoro, A combinatorial interpretation of the recurrence $f_{n+1}=6 f_{n}-f_{n-1}$, Discrete Math., 190 (1998) 235-240.

[2] J. Bonin, L. Shapiro, and R. Simion, Some $q$-analogues of the Schröder numbers arising from combinatorial statistics on lattice paths, J. Statistical Planning and Inference 34 (1993) 35-55.

[3] D. Foata and D. Zeilberger, A classic proof of a recurrence for a very classical sequence, J. Comb. Theor., Ser. A., 80 (1997), 380-384.

Available at http://www.math.temple.edu/ ${ }^{\sim}$ zeilberg/papers1.html.

[4] G. Kreweras, Aires des chemins surdiagonaux a étapes obliques permises. Cahier du B.U.R.O. 24 (1976) 9-18.

[5] A. Martin, Diophantine analysis (Solutions of Problems), Am. Math. Monthly, 4 (1897) 24-25.

[6] D. Merlini, R. Sprugnoli, and M. C. Verri, The area determined by underdiagonal lattice paths, Discrete Math., to appear.

[7] L. Moser and W. Zayachkowski, Lattice paths with diagonal steps, Scripta Math., 26 (1963) 223-229.

[8] M. Newman, D. Shanks and H. C. Williams, Simple groups of square order and an interesting sequence of primes, Acta Aritmetica XXXVIII (1980) 129-140.

[9] E. Pergola and R. A. Sulanke, Schröder triangles, paths, and parallelogram polyominoes, Journal of Integer Sequences, Vol. 1 (1998), Available at http://www.research.att.com/ ${ }^{\sim}$ njas/sequences/JIS/

[10] J-L Rémy, Un procédé itératif de dénombrement d'arbres binaires et son application à leur génération aléatpoire, RAIRO Inform. Théor., vol 19 (1985) 179 195.

[11] D. G. Rogers, A Schröder triangle, Combinatorial Mathematics V: Proceedings of the Fifth Australian Conference. Lecture Notes in Mathematics, vol 622, SpringerVerlag, Berlin (1977) 175-196.

[12] D. G. Rogers, The enumeration of a family of ladder graphs part II: Schröder and superconnective relations, Quart. J. Math. Oxford (2) 31 (1980) 491-506. 
[13] D. G. Rogers and L. Shapiro, Some correspondences involving the Schröder numbers, Combinatorial Mathematics: Proceedings of International Conference, Canberra, 1977. Lecture Notes in Math. 686, Springer-Verlag (1978) 267-276.

[14] E. Schröder, Vier kombinatorische probleme, Z. Math. Phys. 15 (1870) 361 - 376.

[15] R. P. Stanley, Enumerative Combinatorics, Vol. 2, Cambridge University Press, 1999 (tentative)

[16] R. P. Stanley, Hipparchus, Plutarch, Schröder, Hough, Am. Math. Monthly, 104 (1997) $344-350$.

[17] R. A. Sulanke, A recurrence restricted by a diagonal condition: generalized Catalan array, Fibonacci Q., 27 (1989) 33 - 46.

[18] R. A. Sulanke, Three recurrences for parallelogram polyominoes, J. of Difference Eq. and its Appl., (1998 tentative) Available at http://diamond.idbsu.edu/ ${ }^{\sim}$ sulanke/recentpapindex.html

[19] W-J Woan, L. Shapiro, and D. G. Rogers, The Catalan numbers, the Lebesgue integral and $4^{n-2}$, Am. Math. Monthly, 104 (1997) 926 - 931. 